Revue d'histoire de l'Amérique française

REVUE D.HISTOIRE DE L'AMÉRIQUE FRANÇAISE

\title{
L’alphabétisation de la population de la ville de Québec de 1750 à 1849
}

\section{Michel Verrette}

Volume 39, numéro 1, été 1985

URI : https://id.erudit.org/iderudit/304327ar

DOI : https://doi.org/10.7202/304327ar

Aller au sommaire du numéro

Éditeur(s)

Institut d'histoire de l'Amérique française

ISSN

0035-2357 (imprimé)

1492-1383 (numérique)

Découvrir la revue

Citer cet article

Verrette, M. (1985). L'alphabétisation de la population de la ville de Québec de 1750 à 1849. Revue d'histoire de l'Amérique française, 39(1), 51-76.

https://doi.org/10.7202/304327ar d'utilisation que vous pouvez consulter en ligne.

https://apropos.erudit.org/fr/usagers/politique-dutilisation/ 


\title{
L'ALPHABÉTISATION DE LA POPULATION DE LA VILLE DE QUÉBEC DE 1750 A $1849^{1}$
}

\author{
MICHEL VERRETTE \\ Collège Laflèche \\ Trois-Rivières
}

\section{Introduction}

Il y a un peu plus d'une dizaine d'années maintenant que des historiens du Québec et de l'Ontario ont recommencé à s'intéresser au phénomène de l'alphabétisation au cours des siècles passés. Cependant, à notre point de vue, il y a deux questions théoriques qui n'ont pas encore été abordées de manière satisfaisante. D'une part, il y a la question des sources. Quelles sont les sources les plus appropriées pour l'étude de l'alphabétisation aux 17e, 18e et 19e siècles? Dans un bilan provisoire, Allan Greer utilise des résultats provenant de sources aussi diverses que les registres d'état civil, les pétitions, l'enquête Buller et les recensements ${ }^{2}$. Pour nous, il est important de faire la critique des sources disponibles car il est évident qu'elles n'offrent pas toutes la même richesse pour l'étude de l'alphabétisation.

Après l'analyse des sources, nous arrêterons notre réflexion sur la signification du critère de la signature retenu par les historiens dans l'étude de l'alphabétisation. Car si l'on excepte les recensements, toutes les sources envisagées le sont en fonction de la présence ou non de la signature des principaux individus concernés par la source utilisée. Il est donc très important, nous semble-t-il, de définir la valeur et la signification de la signature. Dans une dernière partie, nous présenterons les résultats de notre propre recherche pour la ville de Québec durant la période de 1750 à 1849 .

\section{1 - Les sources}

La lecture des études locales et étrangères sur l'alphabétisation montrent que celles-ci peuvent être menées à partir d'une grande variété de sources: registres judiciaires des dépositions des détenus et des témoins, actes notariés (testaments, contrats de mariages, contrats

\footnotetext{
1 Nous tenons à remercier Monsieur Claude Galarneau, professeur à l'Université Laval, pour ses précieux conseils et la révision du manuscrit de cet article. Le contenu de celui-ci n'engage cependant que son auteur.

Allan Greer, «L'alphabétisation et son histoire au Québec - État de la question», dans Y. Lamonde, L'imprimé au Québec. Aspect historique (18e-20e siècles) (Québec, Institut québécois de recherche sur la culture, 1983), 25-51.
} 
d'apprentissage, etc.), pétitions, listes d'examens des classes de catéchisme, listes des recrues de l'armée, recensements et, finalement, registres d'état civil de mariages. Comme nous le constatons, les sources utilisées ont été nombreuses, variées et, surtout, d'inégale valeur en regard des besoins auxquels elles doivent répondre pour l'étude du phénomène de l'alphabétisation, soit son développement dans le temps et sa pénétration sociale.

\section{Registres judiciaires, listes d'examen, recrues de l'armée, actes notariés}

Nous ne nous attarderons pas sur ces sources puisqu'elles ne s'appliquent que peu ou pas du tout au cas du Québec et qu'elles présentent, à notre point de vue, un caractère trop aléatoire. Cependant, il semble qu'à l'expérience, ces sources puissent constituer une bonne documentation d'appoint et permettre de mieux connaître certains groupes de la population.

\section{Pétitions}

Dans bon nombre de livres d'histoire du Québec, lorsqu'on veut illustrer le degré d'instruction des Québécois au début du 19e siècle, on se réfère à la célèbre pétition de 1827 présentée à lord Dalhousie qui fit de nos ancêtres les «Knights of the Cross». A part propager de tels clichés, de quelle utilité peuvent être les pétitions?

Celles-ci sont d'utilité très limitée parce que, tout d'abord, elles ont un caractère circonstancié et limité géographiquement: "Très nombreux au $19 \mathrm{e}$ siècle, ces documents étaient soumis au gouvernement, par les citoyens d'une localité pour réclamer la nomination d'un juge, pour rendre hommage à un gouverneur, pour affirmer leur loyauté envers la Couronne. $\gg^{3}$ En second lieu, la population circonscrite par les pétitions est elle aussi limitée. Il s'agit généralement d'hommes propriétaires de biens fonciers. On laisse donc de côté toutes les femmes et les jeunes gens en âge d'avoir reçu une instruction mais qui sont non propriétaires. De plus, la pétition ne nous permet que d'établir la proportion d'alphabétisés par rapport au nombre total de pétitionnaires. Finalement, l'organisation de la collecte des pétitionnaires n'est pas très claire. Par exemple, le fait de retrouver la signature de noms de la même main avec, à côté, une croix pose un certain nombre d'interrogations quant aux conditions matérielles liées à l'écriture à cette époque ${ }^{4}$ et au partage des vrais et des faux chevaliers de la croix.

$\mathrm{Au}$ mieux, les pétitions pourraient servir d'élément comparatif, dans une perspective synchronique, pour un endroit précis et pour un groupe bien déterminé de la population.

3 Ibid., 33.

4 Ibid. 


\section{Recensements}

D'après H. J. Graff, les recensements constituent la source la plus sûre, la plus complète et la plus valable pour l'étude de l'alphabétisation ${ }^{5}$. A. Greer considère aussi cette source comme très fiable: «Par rapport aux sources de signatures, les sources de constatation ont l'avantage d'avoir été conçues pour mesurer les connaissances réelles de la population; elles répondent par exemple aux questions: combien savent lire? Savent écrire? ${ }^{6}$ A première vue cette perspective est difficilement contestable puisque le recensement s'adresse à toute la population et revient tous les dix ans. Cependant, les recensements souffrent de certaines faiblesses, nous semble-t-il. Ainsi, pour l'historien qui étudie le développement de l'alphabétisation, les recensements ne commencent qu'en 1851. Il y en a bien eu avant cette date, mais ils sont peu utiles pour notre propos et laissent ainsi dans l'obscurité une très longue période de l'histoire de l'alphabétisation.

Un autre problème que posent les recensements est que l'on connaît mal le procédé de cueillette des données au $19 \mathrm{e}$ siècle. Est-ce que l'enquêteur-recenseur prenait la peine de vérifier si réellement les sujets étaient instruits, ou non? Exigeait-il seulement une signature du chef de famille ou d'un autre membre de la famille pour authentifier et certifier la déclaration? Pour sa part, $\mathrm{A}$. Greer lève cette hypothèse en affirmant que la marge d'erreur due aux fausses déclarations est d'autant moindre que l'échantillonnage est grand'. Même si l'argument est de poids, il ne nous convainc pas complètement. D'ailleurs, la conclusion de Gagan sur les instructions données aux énumérateurs en 1861 renforce notre septicisme:

Illiteracy. Of all the columns on the 1861 return, this is the least accurate. Some enumerators listed no illiterates in their district. Yet in the very next district the rate of adult illiteracy could be as high as fifty or sixty percent. A blank column is, by definition, an assertion of literacy; since the returns vary so wildly from one enumerator to the next, it must be assumed that some other test, preferably literary, must be employed to verify the accuracy of this column. $^{8}$

De plus, pour ce qui est d'établir des séries, il est assez difficile de tisser un lien continu entre les données à cause des changements décennaux dans la répartition des statistiques:

1861 - nombre de personnes fréquentant l'école

- adultes ne sachant ni lire ni écrire

\footnotetext{
${ }_{5}^{5}$ H. J. Graff, The Literacy Myth, Literacy and Social Structure in the Nineteenth Century City (New York, Academic Press, 1979), 329-333.

6 Loc. cit., 34.

7 Ibid., 34.

8 D. P. Gagan, «Enumerator's Instructions for the Census of Canada 1852 and 1861 », Histoire sociale/Social History, 8,14 (novembre 1974): 357.
} 


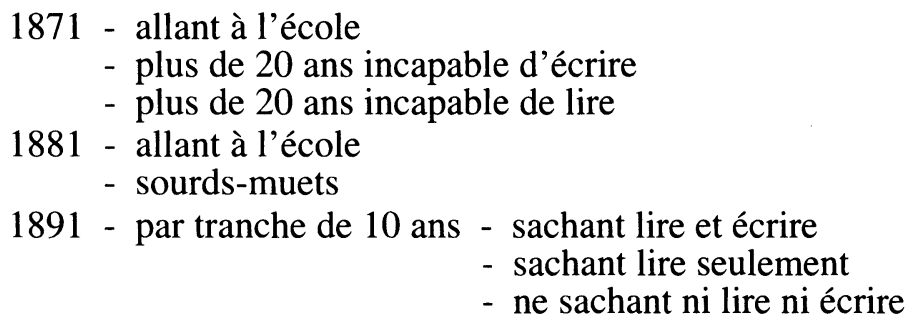

On pourrait conclure que les recensements, utilisés avec beaucoup de précaution, «peuvent constituer une excellente «contre preuve» mais non une source de première main» ${ }^{9}$. Ils peuvent fournir une image fixe, mais non le film de l'évolution de l'alphabétisation.

\section{Actes notariés}

\section{Contrats de mariages}

De toutes les sources disponibles, la plus méconnue est celle des contrats de mariages car jusqu'à présent, à notre connaissance, aucune étude n'a utilisé systématiquement cette source. Cependant, certains indices nous laissent penser qu'ils pourraient constituer une source fort importante ou, à tout le moins, une source de comparaison fiable. Entre autres, il serait intéressant de pouvoir confronter les résultats d'enquêtes menées dans les registres d'état civil (acte public) et dans les contrats de mariages (acte privé). Cela permettrait d'apporter un peu plus d'éclairage sur le phénomène des signatures artificielles des registres: c'est-à-dire de ces gens qui s'efforçaient de signer parce qu'ils étaient en public.

En ce qui concerne la présence des contrats de mariage aux siècles passés et la représentativité sociale, les quelques informations que nous possédons donnent beaucoup d'espoir. Ainsi, Louise Dechêne écrit: «La coutume de passer un contrat de mariage devant notaire est générale à Montréal et probablement dans toute la colonie. . ${ }^{10}$ Aussi, d'après un mémoire de maîtrise sur Montréal entre 1750 et 1770, la proportion des jeunes gens passant un contrat de mariage par rapport au nombre de mariages célébrés serait de plus de $95 \%{ }^{11}$. Si ces tendances se vérifient pour l'ensemble du Québec et pour la période allant jusqu'à la fin du 19e siècle, elles confirmeraient la valeur de cette source.

\footnotetext{
9 J. Rewet et Y. Willemans, L'alphabétisation en Belgique: (XVIIle-XIXe siècles) (Louvain, Bibliothèque de l'Université de Louvain, 1978), 65. 1974), 419.
L. Dechêne, Habitants et marchands de Montréal au XVIIe siècle (Montréal, Plon, 1i Y.-J. Tremblay, La société montréalaise au début du Régime anglais, mémoire de maîtrise (histoire), Université d'Ottawa, 1970, cité dans L. Dechêne, op. cit., 419.
} 
Cependant, cette source présente, croyons-nous, une difficulté pratique de cueillette pour le traitement géographique de l'alphabétisation étant donné qu'un notaire dessert souvent plusieurs villages.

\section{Testaments}

Les testaments sont, avec les registres de l'état civil, les sources les plus utilisées jusqu'à présent par les historiens pour étudier le phénomène de l'alphabétisation, et pour l'américain $\mathrm{K}$. Lockridge, il ne fait aucun doute que le testament est le type de document qui présente les meilleures qualités ${ }^{12}$.

Dans l'étude qu'il a menée sur les colonies américaines, Lockridge évalue entre 20 et $50 \%$ le nombre d'hommes qui testent et entre 2 et $5 \%$ celui des femmes ${ }^{13}$. Pour sa part, $\mathrm{M}$. Vovelle, qui s'intéresse à la Provence, arrive aux chiffres de 60 à $80 \%$ des hommes et de 40 à $60 \%$ des femmes en milieu rural, et de 50 à $35 \%$ des adultes dans les villes; le pourcentage allant en décroissant à mesure que l'importance démographique de la ville croît ${ }^{14}$. Un premier problème paraît évident en ce qui concerne la sous-représentation féminine, principalement aux ÉtatsUnis.

Il faut ensuite se demander qui laisse un testament. Dans les colonies américaines, les deux tiers des personnes les plus riches testent. Cette proportion passe à un tiers pour le groupe intermédiaire, et tombe à un quart pour le groupe le plus pauvre ${ }^{15}$. L'échantillonnage à partir des testaments entraîne donc une sur-représentation du groupe supérieur de la société et une sous-estimation du groupe le plus démuni.

Qu'en est-il pour le Québec? Les renseignements que nous possédons sont très fragmentaires mais tout de même indicatifs. MarieAimée Cliche a dénombré 799 testaments pour l'ensemble du gouvernement de Québec sous le Régime français. Ceux-ci «proviennent des deux sexes, de tous les milieux socio-professionnels et géographiques. Les hommes $(63,2 \%$ des testateurs), les citadins $(58,5 \%)$ et les notables $(36,1 \%)$ sont sur-représentés» ${ }^{16}$. Le faible nombre de testaments et la sur-représentation des notables sont dus au fait que la Coutume de Paris, qui s'appliquera jusqu'à la fin du $18 \mathrm{e}$ siècle, «prévoyait un partage

\footnotetext{
12 K. Lockridge, «L'alphabétisation en Amérique, 1650-1800», Annales ESC, 32,3 (maijuin 1977): 504.

${ }_{13} \mathrm{~K}$. Lockridge, Literacy in Colonial New-England, an Enquiry into the Social Context of Literacy in the Early Modern West (New York, W. W. Norton and Co, 1974), 109.

${ }_{14}$ M. Vovelle, «Maggiolo en Provence: peut-on mesurer l'alphabétisation au début du XVIIIe siècle?», dans «Le XVIIIe siècle et l'éducation», supplément au numéro 88 de la Revue Marseille, ler trimestre (1972): 56.

15 K. Lockridge, loc. cit., 508.

16 M.-A. Cliche, «Les attitudes devant la mort d'après les clauses testamentaires dans le gouvernement de Québec sous le Régime français», Revue d'histoire de l'Amérique française, 32,1 (juin 1978): 59-60.
} 
rigoureux des biens entre les membres de la famille. Les testateurs étaient limités dans le choix de leurs légataires et dans la quantité de biens dont ils pouvaient disposer $(\ldots)^{17}$

Comme nous venons de le voir à partir d'exemples concrets, les testaments présentent des difficultés d'échantillonnage global et social. Nous demeurons donc sceptique quant à la valeur de ce type de sources pour l'étude du développement de l'alphabétisation au Québec.

\section{Registres d'état civil}

S'il ne fait aucun doute pour Lockridge que les testaments constituent la meilleure source pour l'étude de l'alphabétisation, pour J.-G. Daigle, ce sont les registres de mariages qui sont les plus adéquats parce que nous possédons des séries à peu près complètes et qu'ils fournissent un meilleur échantillon de la population adulte ${ }^{18}$.

La dimension sérielle des registres d'état civil ne fait aucun doute. La tenue de tels registres commence en France et en Italie au $14 \mathrm{e}$ siècle. Cette pratique ne se généralise toutefois qu'au $17 \mathrm{e}$ siècle, bien que dès 1563, le Concile de Trente ait prescrit aux curés l'obligation de tenir de tels registres. Pour le Québec, il est possible de remonter jusqu'au début de la colonie ${ }^{19}$. Seuls des accidents de parcours, tel un incendie, et la mauvaise conservation des documents peuvent venir, occasionnellement, altérer ces séries ${ }^{20}$.

Mais en ce qui a trait au problème de la représentation de la population, les registres sont plus représentatifs que les autres sources. Alors que le testament n'est laissé, très souvent, que par les personnes qui ont un certain bien à léguer et qui veulent régler le partage de leur héritage avant de mourir, le pourcentage de la population rejointe par les registres de mariages peut être estimé à $90 \%$ de la population adulte, également répartie entre les deux sexes.

Par contre, une difficulté que semblent poser les registres d'état civil est celle des veufs et des veuves qui contractent une nouvelle union; ce qui peut altérer la validité des statistiques. Ce problème n'en est pas véritablement un puisque au $17 \mathrm{e}$ siècle les veuves comptent pour

\footnotetext{
17 Ibid. Voir aussi Y.-F. Zoltvany, «Esquisse de la Coutume de Paris», Revue d'histoire de l'Amérique française, 25,3 (décembre 1971): 365-384.

18 J.-G. Daigle, «Alphabétisation et culture populaire dans l'Angleterre victorienne: État de la question», Histoire sociale/Social History, 10,19 (mai 1977): 8.

19 G. Bouchard et $\mathrm{A}$. Larose, «La règlementation du contenu des actes de baptêmes, mariages, sépultures, au Québec, des origines à nos jours", Revue d' histoire de l'Amérique française, 30,1 (juin 1976): 68-69. Voir aussi, A. Larose, Les registres paroissiaux au Québec avant 1800: introduction à l'étude d'une institution ecclésiastique et civile (Québec, Ministère des Affaires culturelles, 1980), $298 \mathrm{p}$.

20 C. Veilleux, L'évolution de l'alphabétisation dans le comté de Portneuf, 1690-1849, mémoire de maîtrise (histoire), Université Laval, 1981, 32.
} 
$15,7 \%$ des mariées et les veufs pour $4,3 \%$ des mariés ${ }^{21}$. Pour le seul exemple que nous ayons aux $18 \mathrm{e}$ et $19 \mathrm{e}$ siècles - Sorel -, les veuves, et les veufs représentent respectivement $7,6 \%$ et $10,4 \%$ de la cohorte des nouveaux conjoints ${ }^{22}$. Cet écueil des remariages peut être encore plus limité par l'utilisation de la méthode de l'échantillonnage.

Étant donné les critiques que nous venons de formuler au sujet des différentes sources susceptibles d'être utilisées pour l'étude du développement de l'alphabétisation, nous avons décidé, quant à nous, de privilégier les registres d'état civil. Nous pensons que les avantages de représentation sociale et de disponibilité de longue durée en font la meilleure source. Notre choix des sources étant arrêté, nous aimerions proposer quelques éléments de critique interne des registres d'état civil.

\section{Critique interne des registres d'état civil}

Les registres doivent théoriquement nous fournir les renseignements suivants: la date du mariage, le nom des époux, leur âge, la profession de chacun, le nom des parents des époux et leur profession, le lieu de résidence des époux et celui des parents, la liste des témoins, les signatures des époux et des témoins, s'il y a lieu, et celle du curé. L'acte compte aussi un certain nombre d'autres renseignements qui concernent plus directement la pratique religieuse ${ }^{23}$.

Cela aurait été merveilleux si tous les curés s'étaient conformés rigoureusement aux procédures prescrites. Malheureusement, il n'en va pas toujours ainsi. La plus forte lacune se trouve dans la mention des occupations. On en retrouve très peu avant que l'Assemblée du Bas-Canada n'adopte, en 1795, une loi pour contraindre les curés à indiquer l'occupation soit de l'époux ou de son père. Notons que, même après cette date, on ne mentionne qu'exceptionnellement l'occupation de l'épouse. Par contre, on donne régulièrement celle de son père. Cela est certainement dû au statut social de mineure de la femme.

Un autre exemple d'imprécision est celui de l'âge des époux. L'utilisation fréquente des expressions «majeur» et «mineur» nous empêche d'établir des distinctions valables entre groupes d'âge. Ce problème se complique d'autant plus que du $17 \mathrm{e}$ siècle jusqu'à la fin du $19 \mathrm{e}$ siècle, l'âge où l'on atteint la majorité n'est pas toujours le même.

Le dernier point concerne la cueillette même des signatures des époux. Le curé se doit normalement d'indiquer clairement, par l'une ou l'autre des formules suivantes, la capacité de signer des conjoints:

\footnotetext{
21 L. Dechêne, op. cit., 107.

22 A. Greer, «The Pattern of Literacy in Quebec 1745-1899», Histoire sociale/Social History, 11,22 (novembre 1978): 298

${ }_{23}$ G. Bouchard et A. Larose, loc. cit., et A. Larose, op. cit.
} 
«les deux ont déclaré ne savoir signer», «seul l'époux a su signer», «seule l'épouse a su signer», «les deux ont signé». Selon les démographes de l'Université de Montréal, l'aptitude à signer n'est indiquée que dans un peu plus de $40 \%$ des actes au $17 \mathrm{e}$ siècle (pour la période 1680-1700 elle monte à $65 \%)^{24}$. De son côté, P. Hamelin constate pour la Côte-du-Sud, aux $17 \mathrm{e}$ et $18 \mathrm{e}$ siècles, qu'il y a plusieurs lacunes: «Parfois les curés écrivent que les époux ont signé, mais il n'y a aucune trace de leur signature dans les actes. Plus couramment, le prêtre signe lui-même à la place des époux.» ${ }^{25}$

L'explication de ce phénomène se trouve dans l'organisation même de la desserte des paroisses par les curés. Il arrive souvent à cette époque qu'un même curé soit appelé à desservir plusieurs paroisses. Dans ce cas, il est probable qu'il n'apporte pas toujours avec lui les registres de ces paroisses. Les actes sont pris en note sur un bout de papier: parfois peut-être de mémoire, et, de retour au presbytère de sa paroisse de résidence, le curé consigne les renseignements dans les registres. De ce fait, on retrouve très peu de signatures qui ne sont pas de la main du curé $^{26}$. Parfois le curé ne prend même pas la peine de faire signer ceux qui ont déclaré savoir le faire. Au moment de la cueillette de l'information, il est donc important de tenir compte de cette situation. Par exemple, si le curé dit que les époux ont signé mais qu'il n'y a pas de signature, nous croyons qu'il faut procéder comme s'il y en avait. Car l'absence des signatures peut relever d'une foule de facteurs qui sont hors de la volonté des époux.

En fait, on pourrait résumer l'ensemble de notre analyse des sources pour l'étude de l'alphabétisation et le choix que nous avons fait en disant que nous avons cherché à trouver parmi les documents disponibles portant signature, du $17 \mathrm{e}$ au $19 \mathrm{e}$ siècles, celui qui était le moins déformant de la société ${ }^{27}$.

\section{2 - Le critère de la signature}

Dans le domaine de l'étude du développement de l'alphabétisation aux siècles passés, il est impossible d'interroger les individus. C'est pourquoi il faut bien se tourner vers la source indirecte que sont les signatures. Le problème qui se pose alors est de savoir ce que représente ce critère pour juger du niveau d'alphabétisation.

Tout d'abord, il faut établir et accepter le caractère approximatif des résultats des recherches sur l'alphabétisation. Ce que nous cher-

\footnotetext{
${ }^{24}$ R. Roy et H. Charbonneau, «Le contenu des registres paroissiaux canadiens du XVIIe siècle», Revue d' histoire de l'Amérique française, 30,1 (juin 1976): 89.

${ }_{25}$ P. Hamelin, L'alphabétisation de la Côte-du-Sud, 1680-1869, mémoire de maîtrise (histoire), Université Laval, 1982, 38.

26 A. Gosselin, L'instruction au Canada sous le Régime français, (1635-1760) (Québec, Laflamme et Proulx, 1911), 27.

27 J. Rewet et Y. Willemans, op. cit., 62.
} 
chons, c'est un ordre de grandeur dans la mesure du développement du phénomène. Il importe peu par exemple qu'en 1825 le taux précis d'alphabétisation ait été de $26,5 \%$ et non de $24 \%$ ou de $29 \%$. Par contre il est très important de constater qu'il était autour de $25 \%$ et non de $15 \%$ ou de $45 \%$.

Une deuxième remarque s'impose: quand nous parlons de l'étude de l'alphabétisation, il ne s'agit pas de savoir si nous avons affaire à une société d'intellectuels ou si les gens étaient en mesure d'écrire à la rédaction du journal local pour exprimer leur opinion. Notre but est beaucoup plus modeste que cela. L'étude de l'alphabétisation aux siècles passés vise premièrement à atteindre la connaissance «des niveaux de culture élémentaire» de la population ${ }^{28}$ et, deuxièmement, à «l'analyse d'un des phénomènes capitaux de la civilisation moderne: l'accès de toute une société à la culture savante» ${ }^{29}$, c'est-à-dire du passage d'une société de tradition orale à une société axée sur l'écriture. Comme l'écrit J.-P. Hautecoeur dans son étude Analphabétisme et alphabétisation au Québec, il faut avoir une définition de l'alphabétisation qui colle à la société qu'on étudie et au temps où on l'observe. Les normes qui s'appliquent à notre société ne peuvent être transposées intégralement dans le passé ${ }^{30}$. C'est pour cette raison aussi que nous ne pouvons pas donner de définition figée de ce qu'est l'alphabétisation aux siècles passés. Ce qui est vrai pour la fin du $19 \mathrm{e}$ siècle ne l'est pas nécessairement pour le $17 \mathrm{e}$ ou le $18 \mathrm{e}$ siècle.

C'est dans ces limites très précises que nous devons considérer l'approche du choix des sources. Bien que le critère de la signature soit de plus en plus reconnu comme un bon indicateur, l'unanimité n'est pas encore faite chez les chercheurs. Par exemple, pour Y. Castan qui a étudié la société du Languedoc, il y a «trop d'épreuves négatives» qui entourent la signature pour permettre de mesurer le niveau d'alphabétisation à partir de cette base documentaire ${ }^{31}$. A défaut de connaître les «épreuves négatives» dont fait mention Y. Castan, voyons ce qui tendrait à faire reconnaître le critère de la signature comme étant un bon indicateur.

En français, nous employons le terme alphabétisation pour désigner les recherches sur le développement de l'écriture. Cette expression diffère beaucoup dans son acceptation de la traduction du terme literacy employé par les Anglais. Celui-ci se rapporte au développement de la

\footnotetext{
28 M. Vovelle, loc. cit., RHMC, 89.

29 F. Furet et J. Ozouf, Lire et écrire, l'alphabétisation des Français de Calvin à Jules Ferry (Paris, éd. de Minuit, 1977), 18.

30 J.-P. Hautecoeur, Analphabétisme et alphabétisation au Québec (Québec, Ministère de l'Éducation, Service général des communications, 1979), 10.

${ }_{31}$ Y. Castan, Honnêteté et relations sociales en Languedoc, 1715-1780, dans Jean Queniart, «Les apprentissages scolaires élémentaires au XVIIle siècle: faut-il réformer Maggiolo?», RHMC , 24,19 (janvier-mars 1977): 4.
} 
qualité et de la capacité de lire et d'écrire. Comme l'écrit un des pionniers de l'étude de l'alphabétisation:

Throughout this paper the word «literacy» should be understood to mean capacity to sign one's name, which for periods before the nineteenth century is nearly all we now know or indeed are ever likely to know in the future. We do not know now, and may never know, the precise relationship between the capacity to sign one's name - «alphabetism» might be a better word for it - and true literacy, that is the ability to use the written word as a means of communication. ${ }^{32}$

La capacité de signer se situerait donc quelque part entre l'analphabétisme complet et une connaissance de la lecture et de l'écriture qui permet de communiquer à travers ces deux médias.

En acceptant ce schéma du passage de la civilisation de l'oral à la civilisation de l'écrit, nous constatons que dans un premier temps se développe la lecture, phase de déchiffrage du code qu'est l'alphabet, ensuite vient l'apprentissage de l'écriture, où les gens apprennent à transcrire avec le code alphabétique les mots du vocabulaire; en commençant par les plus simples et ceux qu'ils entendent le plus souvent ${ }^{33}$. Dans ces conditions, quoi de plus normal que d'apprendre à écrire son nom avant toute chose.

Quand on y regarde de près, la hiérarchisation des différents apprentissages scolaires élémentaires (lecture, écriture, calcul) suit le même schéma que la civilisation en fonction de la demande qui vient de la population. C'est sans doute pour cette raison que, souvent, les écoles présentent des programmes permettant de maîtriser successivement et séparément chacun des apprentissages élémentaires ${ }^{34}$.

Partant de la différence de définition entre «alphabétisation» et «literacy» et de la dissociation entre l'apprentissage de la lecture et de l'écriture, que penser de l'indicateur signature? Dans la perspective de l'étude du passage d'une société de type oral à une société basée sur l'écrit, la capacité de signer doit être considérée comme un indicateur qui surestime probablement le nombre de gens capables d'écrire, qui sous-estime, tout aussi probablement, le nombre capables de lire au moins les choses élémentaires et qui donne une indication assez juste

\footnotetext{
1969): 98 .

L. Stone, «Literacy and Education in England (1640-1900)», Past and Present, 42 (february

33 F. Furet et J. Ozouf, op. cit., 349-369.

34 J. Meyer, «Alphabétisation, lecture et écriture: essai sur l'instruction populaire en Bretagne du XVIe au XIXe siècle», Actes du 95e Congrès national des Sociétés savantes, Reims, 1970, Tome I: 333-344. Nos propres recherches sur les écoles de Québec nous montrent le même phénomène où l'on offre d'enseigner chacune des branches de l'instruction élémentaire pour un prix distinct.
} 
de la proportion de la population qui peut lire couramment ${ }^{35}$. Les signatures seraient donc un bon indicateur pour déterminer le trend de l'évolution de l'alphabétisation.

Une des plus récentes études françaises nous fournit deux éléments d'évaluation très importants de la valeur du critère de la signature. En premier lieu, une analyse factorielle, démarche mathématique dont le but est d'exhiber des facteurs indépendants qui permettent d'expliquer la variance des résultats. Autrement dit, on cherche à savoir quelle sera la meilleure combinaison linéaire des variables. Deuxièmement, une démonstration basée sur l'étude de corrélation: c'est-à-dire qu'on essaie de voir le rapport qui s'établit entre deux facteurs qui varient en fonction l'un de l'autre ${ }^{36}$.

Les résultats obtenus par ces deux tests amènent les auteurs à conclure que la «capacité à signer renvoie donc bien à ce que nous appelons aujourd'hui l'alphabétisation et qui compte lecture et écriture» ${ }^{37}$. L'explication de cette définition contemporaine de l'alphabétisation se trouve dans le fait que les auteurs utilisent des données de la seconde moitié du 19e siècle. Par ailleurs, ceux-ci n'écrivent-ils pas plus loin:

Mais qu'importe! Lire seulement, c'est après tout être alphabétisé.

C'est en tout cas, ainsi qu'on l'entend, très avant la seconde moitié du XIXe siècle: un «illétré», c'est alors celui qui ne sait ni lire ni écrire, et la capacité à lire seulement est bien considérée comme un stade élémentaire, mais décisif, de l'alphabétisation. ${ }^{38}$

Une autre démonstration de la valeur du critère de la signature s'articule autour de l'idée du degré d'aisance de la signature ${ }^{39}$.

Marie-Madeleine Compère, l'auteure de la première étude, établit quatre catégories de qualité calligraphique: 1 - très aisée, 2 - appliquée, bien formée, 3 - maladroite, mais lisible, 4 - mal formée, voir informe. Partant de l'examen de l'organisation des résultats de ses recherches, elle constate que les personnes qui appartiennent à la quatrième catégorie, comptent pour moins de $10 \%$ de l'échantillonnage et elle en conclut:

Cette faiblesse renforce le test des signatures comme révélateur de la connaissance de l'écriture: elle démontre en quelque sorte que, subjectivement, l'individu se situe lui-même soit à l'intérieur, soit

35 R. S. Schofield, «The Measurement of Literacy in the Pre-Industrial England», Literacy in Traditionnal Societies (Cambridge, Cambridge University Press, 1968), 324. Voir aussi K. Lockridge, Literacy in..., 7; L. Stone, loc. cit., 98; et J. Queniart, loc. cit., 6-7.

${ }^{36}$ F. Furet et J. Ozouf, op. cit., 13-27.

37 Ibid., 26.

$38 \quad$ Ibid., 299.

39 M.-M. Compère, «École et alphabétisation en Languedoc aux XVIIe et XVIIIe siècles», dans F. Furet et J. Ozouf, ibid., Tome II, 91ss. et J. Quéniart, loc. cit., 10ss. 
hors du groupe des lettrés et ne cherche qu'exceptionnellement à signer, s'il le sait faire mal. ${ }^{40}$

Pour sa part, J. Quéniart, pour établir sa démonstration, forme six catégories: 1 - B-1 simple croix, 2 - B-2 signatures informes, 3 - B-3 signatures dessinées, correctes, lisibles, 4 - A-3 signatures lentes, appliquées, 5 - A-2 écriture courante, 6 - A-1 belles signatures paraphées. Se référant à ses propres recherches, l'auteur observe que les deux catégories qui regroupent les plus hauts scores sont A-2 et B-1. Ainsi, pour Quéniart, le taux réel d'alphabétisation se situe quelque part entre le seuil minimum obtenu par l'addition de A-1 et A-2, et la différence entre $100 \%$ moins B-1 plus B-2. La moyenne entre les deux résultats représenterait une approximation assez juste du taux d'alphabétisation de la population étudiée.

Avant de terminer sur cette question, nous voudrions aborder, très brièvement, deux objections qui peuvent encore être apportées pour essayer de minimiser la valeur du critère de la signature.

La première est la question des «modèles» de signature que l'individu gardait avec lui. Il semble, qu'effectivement, une telle pratique eut cours à une certaine époque ${ }^{41}$. Personnellement, nous n'avons rencontré nulle part, ni dans nos recherches ni dans nos autres lectures, d'autres mentions de ce phénomène et nous croyons qu'il doit être considéré comme marginal.

La seconde objection porte sur l'abstention de signer que peuvent avoir pratiquée certains des conjoints par égard pour l'autre. Cette objection ne peut se concevoir qu'en regard d'une vision toute contemporaine du rôle de l'écriture. La signature n'a pas la même importance aux siècles passés qu'aujourd'hui:

Qu'importe en effet aux curés, aux familles, que l'époux ou l'épouse paraissent illéttrés dans une société où l'ignorance, souvent majoritaire, n'est en aucune manière une tare honteuse qu'on s'efforce de cacher? Si la signature est fonctionnelle, si elle est de l'ordre du pratique beaucoup plus que de celui du prestige social, les nouveaux époux n'ont aucun besoin, dans cette circonstance où ils sont entourés de témoins officiels et officieux dont la présence authentifie de toute façon leur engagement, de forcer leur talent et personne n'a le moindre intérêt à les y contraindre. ${ }^{42}$

Une autre réfutation ou confirmation de cette hypothèse pourrait être apportée, comme nous l'avons déjà mentionné, en faisant le rapprochement des registres de l'état civil avec les contrats de mariage.

\footnotetext{
40 M.-M. Compère, loc. cit., 91.

41 J. Meyer, loc. cit., 336.

42 J. Quéniart, loc. cit., 8.
} 


\section{3 - L'alphabétisation à Québec de 1750 à $1849^{43}$}

Le choix de la période 1750-1849 comme cadre chronologique tient à trois facteurs. D'abord, le fait de pouvoir faire le point sur l'alphabétisation à la fin du Régime français. Ensuite, en poussant notre étude jusqu'en 1849, cela nous permet de tenir compte de l'implantation de nouveaux groupes ethniques après la Conquête. Enfin, nous voulions une période assez longue pour mener une étude conjoncturelle et pouvoir prendre en considération le développement de la législation scolaire dans le premier tiers du $19 \mathrm{e}$ siècle.

Les résultats de notre recherche s'articulent en deux tableaux. Tout d'abord, nous présenterons un bilan global de l'alphabétisation à Québec entre 1750 et 1849. Dans un deuxième temps, nous passerons d'une analyse structurelle à une analyse conjoncturelle. Dans ces deux approches, nous visons à faire ressortir les caractères généraux de l'alphabétisation des Québécois: dimorphisme sexuel, géographie et répartition sociale et ethnique.

\section{Bilan global et ses nuances}

Les données de notre étude sont calculées à partir des registres d'état civil de mariages pour la ville de Québec, soit: la paroisse NotreDame-de-Québec, dont les registres remontent à 1621, la paroisse SaintRoch, créée en 1829 , et, du côté protestant, English Cathedral, qui ouvre ses registres en 1766 .

Le corpus d'informations regroupe la totalité des mariages pour trois années, prises au hasard, dans chaque décennie et pour chacune des paroisses. Ainsi, nous avons relevé 2824 mariages pour NotreDame-de-Québec, 729 pour Saint-Roch et 821 pour English Cathedral. Ce qui fait un total de 4434 mariages ou 8868 personnes.

Globalement, pour toute la période, 39,9\% des conjoints signent le registre des mariages. Pour les gens de Québec même, le taux d'alphabétisation est de $40,7 \%$. Il n'est que de $29,3 \%$ pour les personnes de l'extérieur; principalement des gens des campagnes environnantes.

Le dimorphisme sexuel dans le développement de l'alphabétisation est un aspect important, car il marque un premier clivage social dans la pénétration de ce monde secret qu'est l'écriture. La répartition par sexes montre qu'il y a une nette démarcation entre les hommes $(48,4 \%)$ et les femmes $(31,5 \%)$. Tout près de 17 points séparent ces deux catégories.

\footnotetext{
43 Les données de cette partie sont tirées de notre mémoire de maitrise. Michel Verrette, L'alphabétisation de la population de la ville de Québec, 1750-1849, mémoire de maîtrise (histoire), Université Laval, 1979, 138 p.
} 
La dimension ethnique de l'alphabétisation ne saurait être ignorée au Québec. Dans l'ensemble, les anglophones avec 53,9\% d'alphabétisés sont nettement en avance sur les francophones, qui n'en comptent que $31,9 \%$, soit 12 points de plus pour les Anglais. Cet écart à l'avantage des anglophones est d'autant plus important, du point de vue socioculturel, que pour la période entre 1795 et 1840 ils comptent, en moyenne, pour seulement 29,3\% de la population de Québec $^{44}$.

Nos résultats nous montrent aussi que les protestants présentent un taux d'alphabétisation moyen de $64,7 \%$ contre $34,3 \%$ pour les catholiques. Si nous combinons les facteurs ethnie et religion, du côté angloprotestant le score s'élève à $66 \%$, celui des franco-protestants à seulement 30\% (il faut dire que l'échantillonnage est restreint à 116 personnes dont une bonne proportion de femmes qui représentent déjà un groupe sous-alphabétisé), les anglo-catholiques sont alphabétisés à $48 \%$ et les franco-catholiques à $34,1 \%$. La combinaison ethnie et religion nous force à constater que si la religion peut être un facteur de stimulation $^{45}$, d'autres facteurs, d'ordre ethnique, entre autres, influencent l'alphabétisation.

A l'occasion de la discussion sur les sources, nous avons vu que la question de l'âge est considérée comme un critère essentiel par rapport à la capacité de signer, qu'il soit dû à la capacité physique en regard de l'âge et de la maladie ou au souvenir plus ou moins lointain de l'apprentissage de l'écriture.

L'information contenue dans les registres de mariages est bien maigre pour apporter une contribution un tant soit peu intéressante à l'éclaircissement de ce problème. Nous trouvons peu de désignations d'âges précises, les curés se contentant d'indiquer si les conjoints étaient «majeurs» ou «mineurs». Cependant, la différence entre les deux groupes est si minime qu'elle doit être considérée comme négligeable. Les personnes dites majeures signent dans une proportion de $35,4 \%$ contre $34 \%$ pour les mineures. Cela démontrerait que les personnes qui se marient se regroupent toutes, ou presque, dans une même catégorie d'âge de quelques années au-dessous ou au-dessus de l'âge de la «majorité».

En partageant nos deux grandes catégories entre garçons et filles, le tableau statistique s'aère. Chez les garçons, $44,7 \%$ des majeurs sont

\footnotetext{
${ }^{44}$ E.H. Dahl et al., La ville de Québec, 1800-1850: un inventaire des cartes et plans (Ottawa, s.é., 1975), 27.

45 L'acquisition des principes de la religion protestante est axée sur la lecture de la Bible. Ce qui en fait un facteur d'incitation à l'apprentissage de la lecture. Par contre, la religion catholique est tournée vers l'apprentissage des règles du catéchisme, ce qui entraîne certainement une proportion de catholiques à apprendre à lire. Mais comme les règles du catéchisme peuvent s'acquérir par répétition - d'ailleurs la racine grecque du mot catéchisme signifie instruction orale ceci en ferait un facteur d'expansion moindre de l'alphabétisation que le protestantisme.
} 
alphabétisés et $36,4 \%$ des mineurs le sont. Chez les filles, le rapport est inversé: $26,1 \%$ des majeures signent contre $32 \%$ des mineures.

On peut essayer d'expliquer ce phénomène par l'hypothèse suivante. L'éducation des filles se fait plus tôt que celle des garçons et ceux-ci sont appelés à utiliser plus ce qu'ils ont appris que ne le font les filles. En effet, chez les garçons, l'éducation ne se ferait, pour un certain nombre, que pendant la période de l'apprentissage d'un métier. C'est du moins une des conclusions auxquelles arrivent J.-P. Hardy et T. Ruddel:

Ce que l'on pourrait qualifier d'instruction proprement dite, à savoir la lecture, l'écriture et l'arithmétique, étaient également prévues dans certains contrats d'apprentissage. L'enseignement du métier était donné à la boutique et on apprenait souvent la lecture et l'écriture à l'école du soir. ${ }^{46}$

Comme l'apprentissage commence généralement entre l'âge de 12 et 19 ans et dure entre 3 et 5 ans, selon les métiers ${ }^{47}$, ceci a pour effet de rapprocher la période de l'apprentissage de l'écriture de celle de la pratique du métier et de la fréquentation de l'écriture, en l'occurrence de la signature du registre de mariage. Par contre, le type d'occupations réservées aux filles et le fait que bien souvent elles restent à la maison pour aider leur mère, les éloigneraient de la pratique de l'écriture. Ainsi, au moment de leur mariage, le souvenir de l'écriture serait moins frais à leur mémoire. On peut examiner aussi le problème sous l'angle des programmes scolaires offerts aux garçons et aux filles. Après avoir exploré le dossier des écoles à Québec entre 1764 et $1859^{48}$, nous avons constaté une forte inclinaison des programmes des écoles de filles vers l'enseignement ménager.

Un autre facteur à considérer dans notre explication est le statut juridique de chacun des deux groupes. Aux 18e et 19e siècles, nous avons affaire à une société bâtie par et pour les hommes, où l'on ne reconnaît que très rarement à la femme une personnalité juridique. Quand on considère les circonstances qui rapprochent les gens de la pratique de l'écriture, ces éléments sont très importants par rapport à leur capacité de signer.

L'aspect principal, à notre point de vue, du développement de l'alphabétisation, est la généralisation progressive d'un modèle culturel dominé par les classes supérieures. Comme l'écrivent Furet et Ozouf: «Partout, toujours, la stratification sociale domine l'histoire de l'alpha-

\footnotetext{
$46 \quad$ J.-P. Hardy et T. Ruddel, Les apprentis artisans à Québec 1660-1815 (Montréal, Presses de l'Université du Québec, 1977), 161.

47 Ibid., 104.

48 Dossier constitué sous la direction de Claude Galarneau.
} 
bétisation. (...) La chronologie du mouvement obéit à sa «descente» dans le corps social.» ${ }^{49}$

La dimension sociale de la pénétration de l'alphabétisation est l'aspect le plus complexe, celui qui exige le plus de souplesse dans son organisation. Nous pensons que la structure d'analyse retenue doit être basée sur des critères tant sociaux qu'économiques, c'est-à-dire que la hiérarchie établie doit tenir compte d'une part du rôle économique dévolu à l'occupation et, d'autre part, de l'estime sociale ou du statut qu'on lui accorde, compte tenu des caractéristiques particulières qui s'y rattachent tels que les qualifications spéciales et le cadre de travail qui l'entoure. Ce travail d'organisation est d'autant plus compliqué qu'on part de la simple mention d'une occupation.

Personnellement, nous avons retenu une hiérarchie qui se divise en trois catégories: supérieure, intermédiaire et populaire. Chacune de ces catégories est ensuite divisée en sous-catégories tels que les professions libérales, le commerce, les artisans, les fonctionnaires, etc. En tout, nous avons retenu treize sous-catégories.

Supérieur

Professions libérales

Affaires

Officiers

Autres
Groupes socio-professionnels

Intermédiaire

Boutiquiers

Artisans autonomes

Sous-officiers

Fonctionnaires

Autres

\section{Populaire}

Artisans

Ouvriers salariés

Soldats

Petits fonctionnaires

La catégorie supérieure est alphabétisée à $87,9 \%$; $92,4 \%$ chez les garçons et $83,3 \%$ chez les filles. Les gens des professions libérales sont alphabétisés à peu près à $100 \%$. C'est la sous-catégorie des gens du commerce qui pèse le plus sur la moyenne générale de la catégorie supérieure avec un taux de $82,9 \%$ d'alphabétisés. Les femmes n'atteignent même pas le plateau de $80 \%(77,4 \%)$. Ajoutons que ce sont les Canadiens qui signent le moins dans cette sous-catégorie avec $86,9 \%$ des non-signataires.

La catégorie intermédiaire marque une baisse importante par rapport à la première avec $55,8 \%$ d'alphabétisation. Le décalage entre les deux est de $32 \%$. Cependant, notre groupe se situe toujours au-dessus de la moyenne générale de $39,9 \%$. Ce qui en fait, du point de vue de l'alphabétisation, un groupe privilégié. Le rapport du nombre de femmes alphabétisés sur le nombre d'hommes est d'un peu plus des deux tiers, comparativement à neuf sur dix pour la catégorie supérieure et à une femme pour deux hommes pour la troisième catégorie. Plus on descend vers la masse, plus l'écart se creuse entre les hommes et les femmes.

$49 \quad$ F. Furet et J. Ozouf, Lire et écrire..., Tome I: 351. 
Reste le groupe des milieux populaires où le taux d'alphabétisation atteint tout juste $31 \%$. Comme ce groupe forme la très grande majorité de la population, on peut conclure que la population de la ville de Québec est faiblement alphabétisée entre 1750 et 1849. Fait très important à noter, à l'intérieur de ce groupe, un écart d'environ $10 \%$ sépare les artisans des journaliers ou des travailleurs sans métier.

Même si l'état des connaissances sur la ville de Québec est limité, on peut tout de même essayer d'établir une cartographie générale de l'alphabétisation de la population de Québec. Ainsi, la haute-ville se diviserait en deux groupes diamétralement opposés: le secteur nord, où l'on retrouve surtout des artisans et des ouvriers, faiblement alphabétisés, et les gens des rues avoisinant la Citadelle, qui appartiennent presque exclusivement aux groupes de la catégorie supérieure les plus fortement alphabétisés. La basse-ville présente sensiblement le même schéma. Cette partie de la ville abrite d'une part des marchands et des aubergistes et, d'autre part, des ouvriers du port. Cependant, dans ce quartier, la ligne de démarcation entre les deux groupes est probablement moins prononcée que pour la haute-ville. Si l'on excepte la partie du faubourg Saint-Jean au-delà de la rue Saint-Jean et du côteau SainteGeneviève, en direction du chemin Saint-Louis qui, de par sa composition sociale, appartient au groupe de population le plus alphabétisé, tout le reste de la population des faubourgs Saint-Jean et Saint-Roch, de loin les plus populeux et regroupant le gros des effectifs des journaliers et des artisans est de loin la partie la plus analphabète de la ville ${ }^{50}$.

Maintenant que nous avons vu comment se présente structurellement le phénomène de l'alphabétisation à Québec, il nous faut examiner le comportement de nos différentes composantes dans une perspective diachronique.

Le tableau des moyennes décennales (tableau 1) est quelque peu énigmatique. Il ne présente pas vraiment une évolution dans le sens d'un progrès ou d'un recul. Les données oscillent continuellement entre 36 et $45 \%$. Nous n'arrivons pas à percevoir une ligne de progression. On croirait avoir affaire à une société en stagnation dont les progrès et les reculs de l'alphabétisation évoluent au rythme des grands événements qui ont marqué la période.

En effet, le point de départ de notre enquête se situe au moment de la guerre de la Conquête. Le score de la décennie 1750-59 concerne donc les jeunes gens qui ont reçu leur instruction avant le début de la

\footnotetext{
50 Pour ce qui concerne les connaissances de la ville de Québec, voir entre autres: E. H. Dahl et al, op. cit.; A. Drolet, La ville de Québec, histoire municipale, tome II et III (Québec, Société historique de Québec, 1965 et 1967); A. Jobin, La petite histoire de Québec (Québec, s.é., 1948).
} 
TABLEAU 1

Population alphabétisée par décennie (\%)

\begin{tabular}{|c|c|c|c|c|c|c|c|c|c|c|}
\hline & $1750-59$ & $1760-69$ & $1770-79$ & $1780-89$ & $1790-99$ & $1800-09$ & $1810-19$ & $1820-29$ & $1830-39$ & $1840-49$ \\
\hline Hommes & 49,5 & 48,1 & 40,9 & 56,7 & 47,8 & 42,6 & 49,6 & 54,0 & 48,2 & 46,0 \\
\hline Femmes & 36,8 & 34,9 & 30,9 & 33,0 & 38,0 & 35,4 & 35,7 & 32,2 & 30,2 & 27,6 \\
\hline Ensemble & 43,1 & 41,5 & 35,9 & 44,9 & 42,9 & 39,0 & 42,7 & 43,0 & 39,2 & 36,8 \\
\hline
\end{tabular}

période des guerres. Ce qui expliquerait, en partie, son taux légèrement supérieur à celui du groupe de la seconde décennie qui comprend une proportion de personnes ayant souffert des inconvénients dus à la guerre.

En regardant l'ensemble du tableau, on remarque que le niveau d'alphabétisation à la fin du Régime français n'est ni plus haut ni plus bas que pour le reste de la période. Il se situe dans la moyenne. Cette situation va à l'encontre de l'hypothèse qui veut que la population de la Nouvelle-France ait eu un niveau assez élevé d'alphabétisation comparé avec celui qui prévaut sous le Régime anglais.

Pour pouvoir vraiment comparer avec les statistiques des autres décennies, une pondération des données de la première décennie est probablement nécessaire vu la présence d'un fort contingent de soldats français, fraîchement débarqués, dans la liste des conjoints. En fait, plus du quart des gens de la première décennie se disent originaires de France.

Ce sont les gens qui se marient entre 1770-79 qui forment la génération du plus grand nombre de gens scolarisables coïncidant avec la période même de la guerre de la Conquête et les années de reconstruction. Il ne faut donc pas s'étonner si cette décennie marque le plus bas niveau d'alphabétisation. Cependant, la reprise ne se fait pas attendre. En 1780-89, on atteint le plus haut score pour le siècle avec $44,9 \%$ d'alphabétisés. Cette décennie marque aussi le progrès le plus important de toute la période, $25,1 \%$. Si la guerre de la Conquête a affecté le développement de l'alphabétisation, ce fut donc, semble-t-il, de courte durée. Ceci nous renvoie à la thèse qui veut que les grands événements politiques n'auraient que peu d'influence marquante et durable sur un phénomène de structure comme l'alphabétisation ${ }^{51}$.

Le deuxième creux de la vague dans le développement de l'alphabétisation s'étend de 1790 à 1809 et est attribuable, pensons-nous, à au

$51 \quad$ F. Furet et J. Ozouf, op. cit., 40, 57. 
moins deux causes. Premièrement, à la guerre d'invasion américaine qui a mobilisé l'activité de la population dans la deuxième moitié de la décennie 1770-79. Ensuite, au retard apporté par les élites canadiennesfrançaises à se redonner de petites écoles après 1760 . La dernière remontée, de 1810 à 1829 , coïncide avec une période de tranquillité sociale et de paix pendant laquelle administrateurs et particuliers s'activent à l'organisation de l'instruction de la population.

Enfin, la baisse des vingt dernières années ne peut s'expliquer, en tout ou en partie, par un événement unique de grande importance. Nous pensons qu'il faut chercher du côté de l'histoire socio-économique de la ville de Québec. Songeons au flot d'émigrants qui y débarquent depuis les années 1820. Québec est devenu un grand chantier de construction navale et le port le plus important de la colonie ${ }^{52}$. Ces transformations rapides ont eu pour effet d'attirer une forte main-d'oeuvre disponible, non qualifiée, originaire des campagnes. Cette hypothèse parait d'autant plausible, si l'on regarde les données de la population de Québec à partir de 1820. (Voir tableau 2)

Dans les paragraphes précédents, nous avons cherché à expliquer les mouvements de l'alphabétisation entre 1750 et 1849 à Québec. Pour

TABLEAU 2

Population de la ville de Québec de 1754 à 1851

\begin{tabular}{|c|c|c|c|}
\hline & Drolet $^{1}$ & Jobin $^{2}$ & En collaboration ${ }^{3}$ \\
\hline 1754 & 8000 & & \\
\hline 1760 & & 8000 & \\
\hline 1765 & 8967 (avec la & 8967 & \\
\hline 1784 & 6491 garnison) & & \\
\hline 1790 & 14000 & 14000 & \\
\hline 1795 & & & 7299 \\
\hline 1805 & & & 8862 \\
\hline 1809 & 16500 & & \\
\hline 1819 & 15527 & & 15257 \\
\hline 1820 & & 15257 & \\
\hline 1831 & 28000 & & 27264 \\
\hline 1832 & 32956 (avec la & & \\
\hline 1840 & garnison) & 26000 & \\
\hline 1844 & 32876 & & 31450 \\
\hline 1851 & 42052 & & 41639 \\
\hline
\end{tabular}

1 A. Drolet, tome II, 14 et tome III, 103.

2 A. Jobin, 118-121

${ }^{3}$ E. H. Dahl, 27

52 E. H. Dahl et al., op. cit., 14-15. 
chaque décennie, ou pour plusieurs, notre tentative d'explication tourne presque toujours autour d'un événement unique ou d'une conjoncture simplifiée, auquel on accorde une importance déterminante. Par exemple, on ne peut minimiser l'impact d'une guerre, surtout pas au $18 \mathrm{e}$ siècle, non plus que le rôle d'une émigration massive ni les effets d'une transformation économique rapide sur la vie d'une communauté de taille somme toute assez réduite.

$\mathrm{Au}$-delà des facteurs conjoncturels, d'autres facteurs d'ordre structurel ont influencé le développement de l'alphabétisation. Que l'on songe à la mentalité des gens. L'alphabétisation, comme n'importe quelle chose, n'est désirée par les gens que lorsqu'ils en ressentent le besoin. Or, une des plus importantes conditions qui créent le besoin de savoir écrire est le niveau de développement économique. Furet et Ozouf affirment que la généralisation de l'alphabétisation vient de l'économie de marché, de la division du travail, et du développement du rôle de l'État qui accompagne ces changements ${ }^{53}$. Le Québec, et la ville de Québec même, sont loin de ce stade de développement économique à la période que nous étudions. Cela, même si la ville connaît un certain développement industriel qui s'organise autour de la construction navale et de quelques industries de transformation du bois. Nous n'avons pas affaire à un mode de production marqué par le capital, le machinisme et la division du travail.

D'autres faits sont aussi à considérer. Par exemple, sous le Régime français, l'instruction est la responsabilité de l'Église. Après 1760, celleci se voit forcée de partager cette responsabilité avec l'État, qui est anglais et protestant. Ce qui eut pour effet d'entraîner le clergé à limiter ses actions concrètes dans la création d'écoles et à élaborer toute une philosophie anti-éducation populaire sous couvert de la protection de la langue et de la religion du peuple ${ }^{54}$.

Enfin, il nous faudrait aussi tenir compte de certains aspects de l'histoire culturelle, tel que celui du développement de la presse périodique (premier journal en 1764, en 1800 il y en a toujours qu'un seul à Québec, en 1820 il y en a quatre, et en 1850 , six). Il serait intéressant de connaître davantage le rapport entre le développement de l'alphabétisation et celui de la presse périodique.

En examinant la question du dimorphisme sexuel (tableau 1) on est frappé par la similarité des données des lignes concernant les hommes et les femmes. La tendance des deux sexes suit à peu près la même

$53 \quad$ F. Furet et J. Ozouf, op. cit., 352.

54 Voir, entre autres, L.-P. Audet, Histoire de l'enseignement au Québec, tome I: 16081840 (Montréal, Holt, Rinehart and Winston, 1971), 432 p. Richard Chabot, Le curé de campagne et la contestation locale au Québec de 1791 aux troubles de 1837-38 (Montréal, Hurtubise HMH, 1975 ) et Nadia Fahmy-Eid, Le clergé et le pouvoir politique au Québec, une analyse de l'idéologie ultramontaine au milieu du XIXe siècle (Montréal, Hurtubise HMH, 1978). 
direction, excepté à la cinquième et à la huitième décennies. Dans le premier cas, comparé à la décennie précédente, alors qu'il y a baisse marquée du niveau d'alphabétisation chez les garçons, les filles continuent leur progression entreprise vingt ans plus tôt. Comment expliquer ce décalage? Peut-être par la plus lente pénétration de l'alphabétisation chez les filles.

La seconde différence des données présente un phénomène inverse du premier cas. Alors que le score des filles stagne au début du $19 \mathrm{e}$ siècle et qu'après 1819 elles entreprennent une longue et progressive descente qui dure au moins trois décennies, les garçons marquent une progression qui s'étend de 1810 à 1829 , suivie d'un recul de 8 points, égal à celui des filles, dans les deux dernières décennies. Cet autre exemple doit être considéré comme un autre indice de la plus grande lenteur des filles à suivre les aléas du développement de l'alphabétisation. Ce que viendrait encore appuyer les données qui suivent.

La différence d'amplitude des pourcentages des deux groupes est remarquable. Les écarts, à la baisse comme à la hausse, sont beaucoup moins importants chez les filles que chez les garçons. Dans le cas du progrès décennal (tableau 3), la marge s'étire entre $-15,7 \%$ et $38,6 \%$ pour les garçons, et seulement entre $-11,5 \%$ et $15,1 \%$ pour les filles. Pour la différence décennale (tableau 4), peut-être encore plus significative, les écarts vont de $-8,9 \%$ à $15,8 \%$ pour les premiers et de $-4 \%$ à $5 \%$ pour les secondes.

TABLEAU 3

Progrès décennal de l'alphabétisation (\%)

\begin{tabular}{|l|c|c|r|r|r|r|r|r|c|}
\hline & II & III & \multicolumn{1}{c|}{ IV } & V & VI & VII & VIII & IX & X \\
\hline Hommes & -2.8 & -15 & 38.6 & -15.7 & -10.9 & 16.4 & 8.9 & -10.7 & -4.6 \\
Femmes & -5.2 & -11.2 & 6,8 & 15.1 & -6.8 & .8 & -9.8 & -6.2 & -8.6 \\
\hline
\end{tabular}

TABLEAU 4

Différences décennales des scores (\%)

\begin{tabular}{|l|c|c|c|c|c|c|c|c|c|}
\hline & $(2-1)$ & $(3-2)$ & $(4-3)$ & $(5-4)$ & $(6-5)$ & $(7-6)$ & $(8-7)$ & $(9-8)$ & $(10-9)$ \\
\hline Hommes & -1.4 & -7.2 & 15.8 & -8.9 & -5.2 & 7 & 4.4 & -5.8 & -2.2 \\
Femmes & -1.9 & -4 & 2.1 & 5 & -2.6 & .3 & -3.5 & -2 & -2.6 \\
\hline
\end{tabular}


Si nos moyennes décennales d'ensemble nous laissent voir une tendance à la stagnation, la répartition par groupes ethniques (tableau 5) permet d'apprécier l'apport respectif de chacun des deux principaux groupes ethniques au développement de l'alphabétisation des Québécois. On voit très bien que les Canadiens français ne retrouvent jamais plus leur niveau d'alphabétisation d'avant la Conquête. Est-ce à dire que ces nouvelles statistiques viennent remettre en question ce que nous avons dit plus haut sur la Conquête. Nous ne le pensons pas étant donné que nous pouvons noter pour les francophones dans la première moitié de la période le même mouvement que dans la courbe d'ensemble: soit une baisse marquée jusqu'à la troisième décennie, attribuable à la guerre et à ses séquelles, puis une remontée entre 1780 et 1799 . La baisse marquée et durable de l'alphabétisation commence à partir de 1800 , curieusement, juste au moment où les élites se préoccupent le plus d'éducation.

Du côté anglais, les statistiques pour la période 1770-1819 sont assez stables, exception faite du creux de 1780-89. Cependant, à partir de 1820-29 le taux d'alphabétisation des anglophones perd près de 17 points en vingt ans, et la baisse se poursuit encore, quoique plus faiblement, en 1840-49.

Les faits observés dans les deux derniers paragraphes ne permettent pas de remettre en question nos hypothèses quant au rôle de la Conquête dans le développement de l'alphabétisation. Ils nous renvoient plutôt à nos observations sur la qualité socio-culturelle du groupe des migrants de la campagne vers Québec, à partir de 1800, et des immigrants britanniques, majoritairement irlandais, qui arrivent à Québec à partir des années 1820 .

L'étude conjoncturelle du développement de l'alphabétisation selon les groupes socio-professionnels regroupés (tableau 6) n'apporte pas un éclairage nouveau sur le problème de l'alphabétisation. Elle ne fait que

TABLEAU 5

Population alphabétisée selon la nationalité par décennie (\%)

\begin{tabular}{|c|c|c|c|c|c|c|c|c|c|c|}
\hline & $1750-59$ & $1760-69$ & $1770-79$ & $1780-89$ & $1790-99$ & $1800-09$ & $1810-19$ & $1820-29$ & $1830-39$ & $1840-49$ \\
\hline Français & 43,1 & 41,5 & 31,5 & 35,0 & 37,2 & 31,0 & 31,1 & 31,2 & 29,0 & 28,6 \\
\hline Anglais & & & 63,4 & 57,9 & 64,6 & 65,3 & 66,8 & 57,0 & 50,3 & 48,9 \\
\hline
\end{tabular}


TABLEAU 6

Population alphabétisée selon les groupes socio-professionnels regroupés par décennie $(\%)$

\begin{tabular}{|c|c|c|c|c|c|c|c|c|c|c|}
\hline & $1750-59$ & $1760-69$ & $1770-79$ & $1780-89$ & $1790-99$ & $1800-09$ & $1810-19$ & $1820-29$ & $1830-39$ & $1840-49$ \\
\hline $\begin{array}{c}\text { Supé- } \\
\text { rieur }\end{array}$ & 100,6 & 85,7 & 100,0 & 79,5 & 90,5 & 91,4 & 84,4 & 91,7 & 84,6 & 88,5 \\
\hline $\begin{array}{c}\text { Intermé- } \\
\text { diaire }\end{array}$ & 64,6 & 100,0 & 75,0 & 55,9 & 56,8 & 44,3 & 52,6 & 56,5 & 59,2 & 55,7 \\
\hline $\begin{array}{c}\text { Popu- } \\
\text { laire }\end{array}$ & 37,7 & 50,0 & 83,3 & 50,0 & 28,5 & 27,2 & 32,2 & 33,8 & 30,2 & 28,9 \\
\hline
\end{tabular}

confirmer nos conclusions d'ensemble sur la répartition sociale de l'alphabétisation ${ }^{55}$.

Le groupe supérieur a de la difficulté à se maintenir autour de $90 \%$ d'alphabétisation. Cette difficulté, croyons-nous, doit être mise directement en relation avec l'accroissement du nombre de gens dans cette catégorie. A partir des sous-catégories prises séparément (tableau 7), nous constatons que les gens des professions libérales sont tous alphabétisés, du début à la fin, à trois exceptions près. Dans le premier cas, il s'agit d'une jeune fille mineure dont le père est fermier à Saint-Gervais et qui épouse un notaire à l'English Cathedral. Le second cas concerne deux anglophones protestants, un médecin et la veuve d'un cordonnier. Enfin, deux autres anglophones catholiques: le marié est ingénieur et nous ne savons rien de l'épouse.

Dans la catégorie intermédiaire, les boutiquiers et les artisans autonomes sont les deux seuls groupes à marquer un rythme de progression notable. Chez les boutiquiers, les chiffres montrent un accroissement à partir de 1790-99 jusqu'à la fin de la période. Au cours de ces 60 années, le taux de croissance est de $47,4 \%$, passant de $36,7 \%$ à $54,1 \%$ d'alphabétisés. Du côté des artisans autonomes, la croissance est remarquable dans les quatre dernières décennies, mais elle est beaucoup plus faible que pour le groupe précédent, seulement $16 \%$, passant de $70 \%$ en 1810-19 à $81,2 \%$ en 1840-49. La différence de croissance s'explique sans doute par la différence des points de départ de chacune des catégories.

\footnotetext{
55 Nous considérons nos données statistiques comme significatives à partir de la décennie 1790-99. Avant cela, l'échantillonnage avec mention de profession est trop faible.
} 
TABLEAU 7

Population alphabétisée selon les groupes socio-professionnels

\begin{tabular}{|c|c|c|c|c|c|c|c|c|c|c|}
\hline DÉCENNIES & 1750 & 1760 & 1770 & 1780 & 1790 & 1800 & 1810 & 1820 & 1830 & 1840 \\
\hline professions libérales & $\begin{array}{c}4 / 4 \\
100 \%\end{array}$ & & $\begin{array}{c}2 / 2 \\
100 \%\end{array}$ & $\begin{array}{c}4 / 4 \\
100 \%\end{array}$ & $\begin{array}{l}10 / 10 \\
100 \%\end{array}$ & $\begin{array}{l}12 / 12 \\
100 \%\end{array}$ & $\begin{array}{l}19 / 20 \\
95 \%\end{array}$ & $\begin{array}{l}28 / 28 \\
100 \%\end{array}$ & $\begin{array}{c}41 / 42 \\
97.6 \%\end{array}$ & $\begin{array}{c}57 / 58 \\
98.3 \%\end{array}$ \\
\hline commerçants & $\begin{array}{c}6 / 6 \\
100 \%\end{array}$ & $\begin{array}{l}8 / 10 \\
80 \%\end{array}$ & $\begin{array}{c}6 / 6 \\
100 \%\end{array}$ & $\begin{array}{c}19 / 22 \\
86.4 \%\end{array}$ & $\begin{array}{c}19 / 22 \\
86.4 \%\end{array}$ & $\begin{array}{c}42 / 48 \\
87.5 \%\end{array}$ & $\begin{array}{c}41 / 54 \\
75.9 \%\end{array}$ & $\begin{array}{c}60 / 66 \\
90.9 \%\end{array}$ & $\begin{array}{c}104 / 132 \\
78.8 \%\end{array}$ & $\begin{array}{l}83 / 102 \\
81.4 \%\end{array}$ \\
\hline officiers de l'armée & $\begin{array}{l}16 / 16 \\
100 \%\end{array}$ & $\begin{array}{c}2 / 2 \\
100 \%\end{array}$ & $\begin{array}{c}2 / 2 \\
100 \%\end{array}$ & $\begin{array}{c}12 / 18 \\
66.7 \%\end{array}$ & $\begin{array}{c}4 / 4 \\
100 \%\end{array}$ & $\begin{array}{c}4 / 4 \\
100 \%\end{array}$ & $\begin{array}{l}12 / 12 \\
100 \%\end{array}$ & $\begin{array}{c}12 / 14 \\
85.7 \%\end{array}$ & $\begin{array}{l}18 / 20 \\
90 \%\end{array}$ & $\begin{array}{c}4 / 4 \\
100 \%\end{array}$ \\
\hline autres & $\begin{array}{c}2 / 2 \\
100 \%\end{array}$ & $\begin{array}{c}2 / 2 \\
100 \%\end{array}$ & & & $\begin{array}{c}5 / 6 \\
83.3 \%\end{array}$ & $\begin{array}{c}6 / 6 \\
100 \%\end{array}$ & $\begin{array}{l}9 / 10 \\
90 \%\end{array}$ & $\begin{array}{c}1 / 2 \\
50 \%\end{array}$ & $\begin{array}{c}8 / 8 \\
100 \%\end{array}$ & $\begin{array}{l}10 / 10 \\
100 \%\end{array}$ \\
\hline boutiquiers & & & $\begin{array}{c}1 / 2 \\
50 \%\end{array}$ & $\begin{array}{c}3 / 4 \\
75 \%\end{array}$ & $\begin{array}{r}11 / 30 \\
36.7 \%\end{array}$ & $\begin{array}{r}15 / 40 \\
37.5 \%\end{array}$ & $\begin{array}{c}22 / 48 \\
45.8 \%\end{array}$ & $\begin{array}{c}32 / 70 \\
45.8 \%\end{array}$ & $\begin{array}{c}51 / 96 \\
53.1 \%\end{array}$ & $\begin{array}{l}66 / 122 \\
54.1 \%\end{array}$ \\
\hline artisans autonomes & $\begin{array}{c}6 / 8 \\
75 \%\end{array}$ & $0 / 1$ & $\begin{array}{c}2 / 2 \\
100 \%\end{array}$ & $\begin{array}{l}3 / 12 \\
25 \%\end{array}$ & $\begin{array}{c}13 / 16 \\
81.2 \%\end{array}$ & $\begin{array}{c}2 / 8 \\
25 \%\end{array}$ & $\begin{array}{l}21 / 30 \\
70 \%\end{array}$ & $\begin{array}{c}35 / 48 \\
72.9 \%\end{array}$ & $\begin{array}{c}25 / 34 \\
73.5 \%\end{array}$ & $\begin{array}{c}13 / 16 \\
81.2 \%\end{array}$ \\
\hline sous-officiers & $\begin{array}{c}10 / 16 \\
62.5 \%\end{array}$ & & & $\begin{array}{c}6 / 8 \\
75 \%\end{array}$ & $\begin{array}{c}4 / 6 \\
66.7 \%\end{array}$ & $\begin{array}{c}7 / 12 \\
58.3 \%\end{array}$ & $\begin{array}{c}3 / 6 \\
50 \%\end{array}$ & & $\begin{array}{c}3 / 4 \\
75 \%\end{array}$ & $\begin{array}{c}4 / 4 \\
100 \%\end{array}$ \\
\hline fonctionnaires & $\begin{array}{l}10 / 10 \\
100 \%\end{array}$ & & $\begin{array}{c}1 / 2 \\
50 \%\end{array}$ & $\begin{array}{c}5 / 8 \\
62.5 \%\end{array}$ & $\begin{array}{c}8 / 8 \\
100 \%\end{array}$ & $\begin{array}{c}3 / 4 \\
75 \%\end{array}$ & $\begin{array}{l}8 / 10 \\
80 \%\end{array}$ & $\begin{array}{c}19 / 24 \\
79.2 \%\end{array}$ & $\begin{array}{c}14 / 18 \\
77.8 \%\end{array}$ & $\begin{array}{c}10 / 14 \\
71.4 \%\end{array}$ \\
\hline autres & $\begin{array}{c}5 / 14 \\
35.7 \%\end{array}$ & $\begin{array}{c}2 / 2 \\
100 \%\end{array}$ & $\begin{array}{c}2 / 2 \\
100 \%\end{array}$ & $\begin{array}{c}2 / 2 \\
100 \%\end{array}$ & $\begin{array}{l}14 / 28 \\
50 \%\end{array}$ & $\begin{array}{c}20 / 42 \\
47.6 \%\end{array}$ & $\begin{array}{c}25 / 56 \\
44.6 \%\end{array}$ & $\begin{array}{c}26 / 56 \\
46.4 \%\end{array}$ & $\begin{array}{c}35 / 64 \\
54.7 \%\end{array}$ & $\begin{array}{c}43 / 88 \\
48.9 \%\end{array}$ \\
\hline artisans & $\begin{array}{c}1 / 2 \\
50 \%\end{array}$ & $\begin{array}{c}2 / 2 \\
100 \%\end{array}$ & $\begin{array}{c}5 / 6 \\
83.3 \%\end{array}$ & $\begin{array}{c}20 / 28 \\
71.4 \%\end{array}$ & $\begin{array}{c}28 / 80 \\
35 \%\end{array}$ & $\begin{array}{l}71 / 180 \\
39.4 \%\end{array}$ & $\begin{array}{c}122 / 294 \\
41.5 \%\end{array}$ & $\begin{array}{c}159 / 406 \\
39.2 \%\end{array}$ & $\begin{array}{c}219 / 636 \\
34.4 \%\end{array}$ & $\begin{array}{c}301 / 946 \\
31.8 \%\end{array}$ \\
\hline travailleurs non-spécialisés & & & $\begin{array}{c}2 / 2 \\
100 \%\end{array}$ & $\begin{array}{c}10 / 28 \\
35.7 \%\end{array}$ & $\begin{array}{c}17 / 78 \\
21.8 \%\end{array}$ & $\begin{array}{l}22 / 158 \\
13.9 \%\end{array}$ & $\begin{array}{l}40 / 224 \\
17.9 \%\end{array}$ & $\begin{array}{c}128 / 462 \\
27.7 \%\end{array}$ & $\begin{array}{c}210 / 810 \\
25.9 \%\end{array}$ & $\begin{array}{c}235 / 924 \\
25.4 \%\end{array}$ \\
\hline armée & $\begin{array}{c}33 / 88 \\
37.5 \%\end{array}$ & & $\begin{array}{c}3 / 4 \\
75 \%\end{array}$ & $\begin{array}{c}22 / 48 \\
45.8 \%\end{array}$ & $\begin{array}{c}6 / 18 \\
33.3 \%\end{array}$ & $\begin{array}{c}7 / 30 \\
23.3 \%\end{array}$ & $\begin{array}{c}12 / 36 \\
33.3 \%\end{array}$ & $\begin{array}{c}2 / 6 \\
33.3 \%\end{array}$ & $\begin{array}{c}11 / 16 \\
68.7 \%\end{array}$ & $\begin{array}{c}11 / 22 \\
50 \%\end{array}$ \\
\hline petits fonctionnaires & & & & & $\begin{array}{c}2 / 2 \\
100 \%\end{array}$ & & $\begin{array}{c}2 / 2 \\
100 \%\end{array}$ & $\begin{array}{l}10 / 10 \\
100 \%\end{array}$ & $\begin{array}{c}2 / 2 \\
100 \%\end{array}$ & \\
\hline \multicolumn{11}{|l|}{ autres } \\
\hline indéterminés & 35 & 39 & 35 & 35 & 15 & 1 & 1 & 3 & 15 & 25 \\
\hline
\end{tabular}


Reste la catégorie populaire. Sa caractéristique première, comme pour les autres, est la stabilité. Celle-ci s'installe à partir de 1790-99 et jusqu'à la fin de la période la variation n'est jamais supérieure à $5 \%{ }^{56}$.

$\mathrm{Au}$ point de vue individuel, le phénomène le plus intéressant à noter est le recul marqué du niveau d'alphabétisation des artisans dans les dernières décennies, en même temps que l'on observe son rapprochement avec le niveau des travailleurs non-spécialisés (salariés). Cette situation doit être rapprochée du phénomène de création des chantiers de construction navale qui fait en sorte que les propriétaires de ces chantiers ont engagé une main-d'oeuvre nombreuse qui apprend son métier sur le tas et qui se coiffe du titre de la profession apprise.

\section{Conclusion}

Dans toute étude la pertinence de la documentation pour la vérification des hypothèses et la justification des conclusions est de première importance. Aussi, à notre point de vue, la seule source qui réunisse les deux conditions de base, séries homogènes et représentation de l'ensemble de la société, est le registre d'état civil des mariages. Quelques-unes des autres sources constituent une bonne documentation d'appoint.

Dans le cadre de l'étude de l'alphabétisation, la question de la valeur du critère de la signature en tant qu'indicateur de l'alphabétisation est âprement discutée. Nous pensons que toute réflexion sur ce sujet doit avoir comme point de départ la définition de ce que l'on entend par alphabétisation aux siècles passés. Le premier effort pour arriver à cette définition est de nous défaire de notre conception élitiste de l'alphabétisation. Aux siècles où l'écrit n'est pas encore un moyen de communication généralisé, le mot alphabétisation trouve sa signification dans l'expression «savoir lire».

L'analyse des principales variables du développement de l'alphabétisation ne nous est pas apparue très concluante sur plusieurs points. Par exemple, la dimension âge des mariés où l'imprécision des données est beaucoup trop grande pour dégager quelque conclusion valable. La différence entre les hommes et les femmes dans l'accès à l'alphabétisation nous fait observer que les femmes sont alphabétisées à un tiers moins que les hommes. Ce qui surprend le plus, c'est que les courbes de développement ne laissent pas percevoir de remontée des scores des femmes pour réduire progressivement l'écart qui les sépare des hommes. Pour sa part, le schéma de la pénétration sociale de l'alphabétisation correspond au schéma classique: un premier groupe de privilégiés par la fortune, les distinctions et les études, qui est pour ainsi dire complè-

\footnotetext{
56 Pour la catégorie supérieure, la différence maximum est de $10,95 \%$ et la moyenne de $6,2 \%$. Pour la catégorie intermédiaire, on obtient $12,45 \%$ comme maximum et $5,3 \%$ de moyenne.
} 
tement alphabétisé, suivi par le groupe intermédiaire qui a un taux d'alphabétisation supérieur de $16 \%$ à la moyenne générale. Enfin, la masse de la population regroupée dans les milieux populaires, avec à peine $31 \%$ d'alphabétisés.

De toutes les données, la plus surprenante est certainement la constatation de la stagnation de l'alphabétisation de la population de la ville de Québec entre 1750 et 1849 . Au-delà de l'explication première que nous avons donnée de ce phénomène, ce qui retient l'attention, c'est l'analyse du rôle qu'a pu jouer la Conquête sur le développement de l'alphabétisation.

Pour l'étude du phénomène de l'alphabétisation, il nous semble tout à fait impropre de chercher l'explication de son développement sur le long terme par la Conquête. L'alphabétisation n'est pas un phénomène de culture locale ou régionale. Elle appartient à la société globale. L'explication doit plutôt être cherchée à l'aide du modèle anthropologique d'acculturation, qui fait de l'alphabétisation le moteur des transformations, premièrement politiques, deuxièmement démographiques et, en dernier lieu, économiques ${ }^{57}$. Cette conception allant à l'encontre du modèle économiste, qui fait des transformations économiques l'initiateur des transformations politiques et démographiques ainsi que de la hausse du taux d'alphabétisation. Il ne faudrait cependant pas voir dans cette approche plus globale de l'alphabétisation une négation de l'influence de tout phénomène historique local.

L'étude de l'alphabétisation de la population de la ville de Québec peut paraître décevante en ce qu'elle n'apporte que peu de conclusions précises. On est presque toujours au stade des hypothèses. En fait, au départ de notre recherche, nous ne pensions pas arriver à des résultats qui dépasseraient ce à quoi nous sommes arrivé. Nous considérons cette étude comme le premier jalon pour jeter les bases d'une étude plus étendue dans le temps et l'espace.

57 Emmanuel Todd, L'enfance du monde. Structures familiales et développement (Paris, Seuil, 1984), $251 \mathrm{p}$. 